\title{
Radiation Effects in Nano Inverter Gate
}

\author{
Nooshin Mahdavi
}

Sama Technical and Vocational Training College, Islamic Azad University, Tehran Branch, Tehran, Iran

\begin{abstract}
Quantum Cellular Automata (QCA) represents an emerging technology at the nanotechnology level. The effects of space radiations in QCA inverter gate are investigated in this paper. Single Electron Fault (SEF) is a fault which may happen during operation of QCA circuits. A detailed simulation based logic level modelling of Single Electron Fault for QCA inverter gate is represented in this paper. It will be shown that the behaviour of single electron fault in QCA devices is not similar to either previously investigated faults or conventional CMOS logic
\end{abstract}

Keywords Space Radiation, Quantum Cellular Automata, Inverter Gate

\section{Introduction}

The microelectronics industry has improved the integration, the power consumption, and the speed of integrated circuits during past several decades by means of reducing the feature size of transistors. But it seems that even by decreasing the transistor sizes, some problems such as power consumption can't be ignored. Utilizing the QCA technology for implementing logic circuits is one of the approaches which in addition to decreasing the size of logic circuits and increasing the clock frequency of these circuits, reduces the power consumption of these circuits. QCA, which was first introduced by Lent et al.[1], represents an emerging technology at the nanotechnology level. QCA cells have quantum dots, in which the position of electrons will determine the binary levels of 0 and 1 .

Various types of cell misplacement faults may occur during fabrication and manufacturing of QCA devices and circuits. Some of them which have been characterized are cell displacement, cell misalignment, cell omission and cell rotation[2-7].

- A cell displacement is a defect in which the defective cell is misplaced from its original direction.

- A cell misalignment is a defect in which the direction of the defective cell is not properly aligned.

- A cell omission is a defect in which a particular cell is missing compared to the original.

- A cell rotation is a defect in which the defective cell is rotated in its location.

There are some other faults, such as missing or extra dots or/and electrons which may occur in QCA devices and such circuits[2, 3]. Single event effects (SEE) are an example of

* Corresponding author:

mahdavi_n2@yahoo.com (Nooshin Mahdavi)

Published online at http://journal.sapub.org/nn

Copyright $(\underset{2}{ } 2012$ Scientific \& Academic Publishing. All Rights Reserved phenomena which can affect QCA devices and circuits. These types of effects can cause electrons to tunnel outside or inside QCA cells, and therefore some remaining QCA cells may contain zero, one, two, three, four or more electrons. This is the main defect caused by SEEs which may occur for QCA devices and circuits. Considering the QCA structure with two electrons in each cell, we can conclude that defected cells may lead to circuit malfunctioning[8].

Nanoelectronic devices are very sensitive to the space environment radiations. The presence of high-energy particles such as protons, heavy ions and electrons, may lead to many hazardous effects. These effects include reduction in performance or functional disruptions that can affect any system operations. This can make satellites experience shortened lifetime or major failures. The main goal of this paper is to model and characterize the single electron fault in QCA inverter gate. This fault may happen in presence of high-energy particles.

The remainder of this paper is as follows. In Section 2, a brief review of QCA is presented. The radiations in the natural space are explained in Section 3. In Section 4, the effects of single electron fault on QCA inverter gate are investigated. Finally, Section 5 concludes this paper.

\section{Quantum Cellular Automata}

In Quantum Cellular Automata (QCA), a cell contains four quantum dots, as schematically shown in Figure 1. The quantum dots are shown as the open circles which represent the confining electronic potential. Each cell is occupied by two electrons which are schematically shown as the solid dots.

In a cell, the electrons are allowed to jump between the individual quantum dots by the mechanism of quantum mechanical tunnelling but they are not allowed to tunnel between cells. The barriers between cells are assumed 
sufficient to completely suppress intercellular tunnelling.
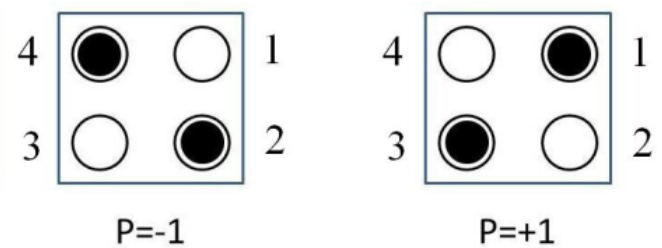

Figure 1. QCA cell and its ground states

If they are left alone, they will meet the configuration corresponding to the physical ground state of the cell. It is in an obvious manner that the two electrons will tend to occupy different dots because of the Coulombic force associated with bringing them together in close proximity on the same dot.

By these concepts, it's concluded that the ground state of the system will be an equal superposition of the two basic configurations with electrons at opposite corners, as shown in Figure 1. The positions of the electrons are also shown in this Figure.

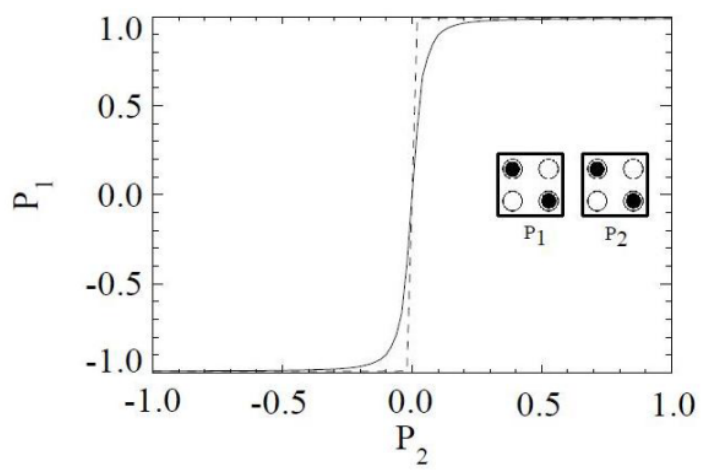

Figure 2. Coupling of QCA cells

Coupling between the two cells is provided by the Coulomb interaction between electrons in different cells. Figure 2 shows how one cell is affected by the state of its neighbour[10]. This figure shows the two cells where the polarization of cell 1 (P1) is determined by the polarization of its neighbour (P2). P2 is assumed to be fixed at a given value, corresponding to a specific arrangement of charges in cell 2 and this charge distribution exerts its influence on cell 1 , thus determining its polarization. The result which can be drawn here is the strongly non-linear nature of the cell-cell coupling. Cell 1 is almost completely polarized even though cell 2 might only be partially and not completely polarized[9, $10]$.

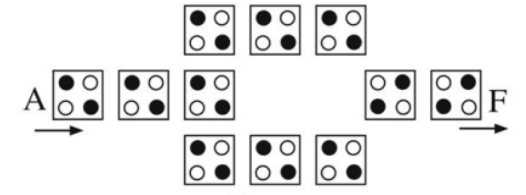

(a)

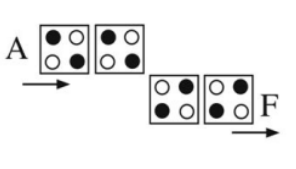

(b)
Figure 3. (a) Redundant inverter gate and (b) Inverter gate

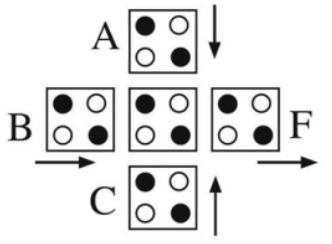

(a)

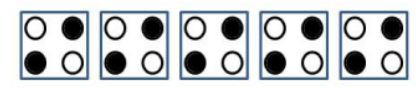

(b)

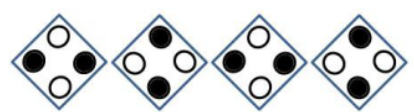

(c)
Figure 4. (a) Majority logic gate, (b) Binary wire, and (c) Inverter chain

The physical interactions between cells may be used to realize elementary Boolean logic functions. The basic logic gates in QCA are the Majority logic function and the Inverter which are illustrated in Figure 4(a) and Figure 3, respectively. The Majority logic function can be realized by only 5 QCA cells[11].

The logic AND function can be implemented by a Majority logic function by setting one of its inputs permanently to 0 and the logic OR function can be implemented by a Majority logic function by setting one of its inputs permanently to 1 .

QCA clocking provides a mechanism for synchronizing information flow through the circuit. It should be considered that the clock also controls the direction of information flow in a QCA circuit. The QCA clock also provides the power required for circuit operation. More precisely, the QCA clock is used to control the tunnelling barrier height in cells. When the clock is low, the electrons are trapped in their associated positions and can't tunnel to other dots, therefore latching the cell (Hold phase). This is caused by the intracellular barriers which are held at their maximum height. When the clock signal is high, the cell goes to the null polarization state (Relax phase). This is caused by the intracellular barriers which are held at their minimum height. Between these two cases, the cells are either releasing or switching.

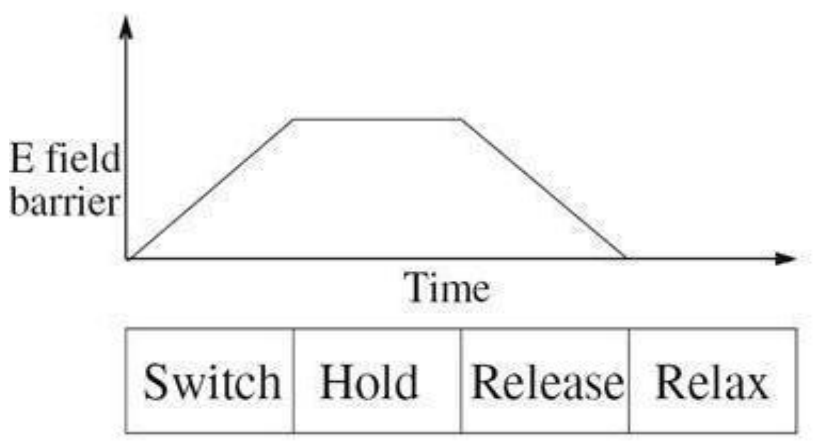

Figure 5. Barrier height in four phases of clock

Figure 5 shows the barrier height in four phases of clock. Each cell in a particular clocking zone is connected to one of the four available phases of the QCA clock shown in Figure 6. Each cell in the zone is latched and unlatched in synchronization with the changing clock signal and therefore the information is propagated through cells[12-15]. 


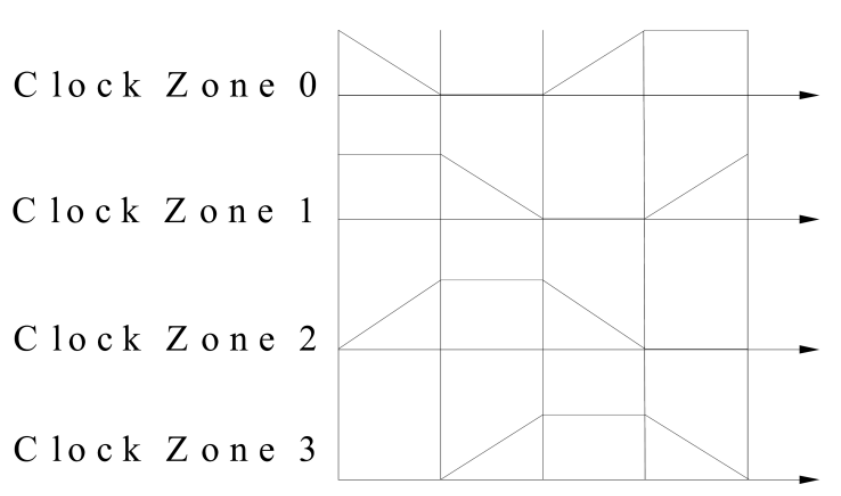

Figure 6. QCA clock zones

\section{Radiation in the Natural Space}

The high-energy ionizing particles of the space environment are responsible for the anomalies observed on electronics.

They can be classified in three categories depending on their origin[16]. Figure 7 illustrates the various space radiations.

The radiation belts: charged particles like electrons (up to $30 \mathrm{MeV}$ ) and protons (up to $500 \mathrm{MeV}$ ) are trapped by the earth magnetic field leading to "stable" and hazardous zones in the near earth vicinity. (The Van Allen belts)

- Solar flares: protons (up to $500 \mathrm{MeV}$ ) and heavy ions in a lesser extend (up to $10 \mathrm{MeV} /$ nucleon) can be emitted during these solar events. The flare occurrence is influenced by the solar cycle: much more event are observed during the so-called "solar maximum" periods.

- The cosmic rays: they are a continuous background of ions whose energy levels can be very high. The origin of cosmic rays lies outside the Solar System. Shock waves and interstellar electromagnetic fields scatter and accelerate ions that can reach thousands of $\mathrm{GeV}$ (significantly higher than those observed during solar flares).

These particles produce various effects when interacting with electronic devices.

Considering a QCA cell, it contains four quantum dots and two electrons which can tunnel between dots. As mentioned before, the barriers between cells are assumed sufficient to completely suppress intercellular tunnelling. References (such as[10]) emphasize that this barrier height is less than $1 \mathrm{eV}$. Considering the ionizing particles which may have several $\mathrm{MeV}$ to several $\mathrm{GeV}$ of energy, this energy can motivate at least 10 million electrons to tunnel outside or inside QCA cells. But according to the size of cells and the effective area of that particle's affection, these motivated electrons are much less. Here we do not want to count the number of electrons which can tunnel outside or inside of QCA cells in presence of an ionizing particle, but we want to investigate the effect of this phenomenon and defects which may occur for QCA cells. After tunnelling of some electrons outside or inside QCA cells, some remaining cells may contain zero, one, two, three, four or more electrons. This is the main defect which may occur for QCA cells and circuits.

\section{Fault Modeling}

In this Section, fault modelling will be accomplished for QCA Inverter gate. All cells are assumed to have a length and width of $18 \mathrm{~nm}$ and quantum dots are $5 \mathrm{~nm}$ in diameter. The centre to centre distance of two neighbour cells is $20 \mathrm{~nm}$. Thus, the cell size can be defined as $20 \mathrm{~nm}$. As an assumption, a $20 \mathrm{~nm}$ cell size was used in[16] and a $25 \mathrm{~nm}$ cell size was used in[5]. Thus, the $20 \mathrm{~nm}$ assumption is valid for QCA cells. The centre to centre distance of two neighbour quantum dots in a QCA cell is $9 \mathrm{~nm}$ (For example, dot1 and dot2 in Figure 1). According to previous definitions, other geometric distances are calculated. As an example, considering two neighbour cells, the distance between dot4 in the left cell and dot 1 in the right cell is $29 \mathrm{~nm}$ and so on.

Also it is assumed that each cell is assigned to an individual clock zone and there are no neighbour cells with the same clock zone. Positions of electrons and polarization of cells are obtained from the fact of the least Kink energy. The Kink energy (electron volt) can be computed by the following equation.

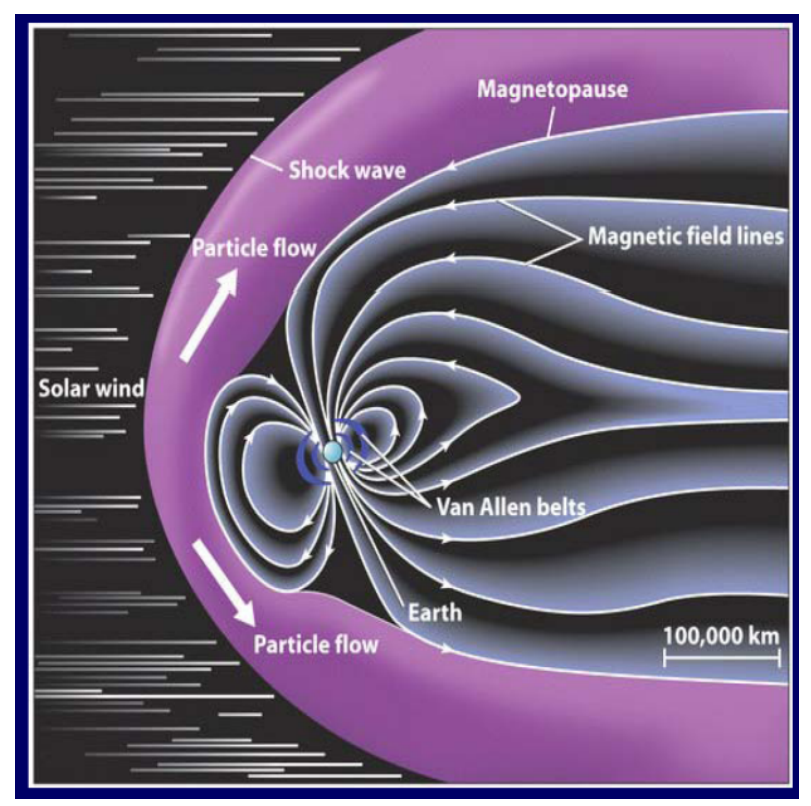

Figure 7. Radiation Belts, Solar Flares, and Cosmic Rays

$$
E_{i, j}=\frac{q_{i} q_{j}}{4 \pi \varepsilon_{0} \varepsilon_{r}\left|r_{i}-r_{j}\right|}
$$

All the following simulations are accomplished by MATLAB software. Now, we will discuss the inverter gate and also redundant inverter gate of Figure 3 which has two inverters in parallel. We have investigated the single electron fault for these two types of inverter gate and modelled the fault for them.

Two major questions should be answered for an inverter gate which contains a faulty cell: 
Considering the faulty cell, where should its single electron go if the previous cell has the polarization of zero or one?

$\checkmark$ Considering the faulty cell, which polarization shall be dictated to its next cell?

For the first case, simulation results show that if a cell is faulty and its previous cell has the polarization of zero, the single electron will go to position number 2 and if its previous cell has the polarization of one, again the single electron will go to position number 2 .

Table 1. Kink energy for positions of electron in a faulty cell in inverter chain according to its previous cell polarization

\begin{tabular}{|c|c|c|c|c|}
\hline $\begin{array}{c}\text { Prev. } \\
\text { Cell Pol. }\end{array}$ & Position 1 & Position 2 & Position 3 & Position 4 \\
\hline Zero & 0.0080519 & 0.0066624 & 0.0080519 & 0.011112 \\
\hline One & 0.0075388 & 0.0063317 & 0.0075388 & 0.0097721 \\
\hline
\end{tabular}

The Kink energy is computed for each position and the position with the least Kink energy is figured out to be the target position. The kink energy of each position is illustrated in Table 1. For the second case, simulation results show that if a cell is faulty and its electron is in positions of 1,2, 3 or 4, the next cell will obtain the polarization of one.

It means that the output is stuck at one. The Kink energy is computed for each polarization and the polarization with the least Kink energy is figured out to be the target polarization. The kink energy of each polarization is illustrated in Table 2 .

Figure 8 illustrates the faulty cell effect on inverter chain. As shown in Figure 8(a), if the left side cell has the logic value of one, the faulty cell will have its electron in position 2 and the right side cell will obtain the logic value of one and as shown in Figure 8(b), if the left side cell has the logic value of zero, the faulty cell will have its electron in position 2 and the right side cell will obtain the logic value of one. According to simulation results, if a single electron fault occurs in an inverter chain, the logic value of that wire will be stuck at one.

Table 2. Kink energy for polarization of next cell in inverter chain according to the position of electron in faulty cell

\begin{tabular}{|c|c|c|}
\hline $\begin{array}{c}\text { Faulty Cell } \\
\text { Position }\end{array}$ & $\begin{array}{c}\text { Next Cell Polarization } \\
\text { Zero }\end{array}$ & $\begin{array}{c}\text { Next Cell Polarization } \\
\text { One }\end{array}$ \\
\hline Position 1 & 0.0080519 & 0.0075388 \\
\hline Position 2 & 0.011112 & 0.0097721 \\
\hline Position 3 & 0.0080519 & 0.0075388 \\
\hline Position 4 & 0.0066624 & 0.0063317 \\
\hline
\end{tabular}

Figure 8. Faulty cell effect on inverter chain

For redundant inverter gate, the fault may occur on 3 cells which are two right most cells on up and down wires and a cell which is at the diagonal neighbourhood of these two cells.
Table 3. Kink energy for positions of electron in a faulty cell in redundant inverter gate according to its previous cells polarization

\begin{tabular}{|c|c|c|c|c|}
\hline $\begin{array}{c}\text { Prev. Cell } \\
\text { Pol. }\end{array}$ & Position 1 & Position 2 & Position 3 & Position 4 \\
\hline Zero & 0.014384 & 0.014201 & 0.017824 & 0.018651 \\
\hline One & 0.014201 & 0.014384 & 0.018651 & 0.017824 \\
\hline
\end{tabular}

Also two previously mentioned questions for the redundant inverter gate should be answered. First, the single diagonal neighbour cell will be investigated. For the first case, simulation results show that if this cell is faulty and its previous up and down cells have the polarization of zero, the single electron will go to position number 2 and if its previous cells have the polarization of one, the single electron will go to position number 1. The Kink energy is computed for each position and the position with the least Kink energy is figured out to be the target position. The kink energy of each position is illustrated in Table 3.

Figure 9 illustrates the output faulty cell effect on redundant inverter gate. Simulation results show that the redundant inverter gate will act as a wire in presence of single electron fault in the output cell, i.e. the single diagonal neighbour cell.

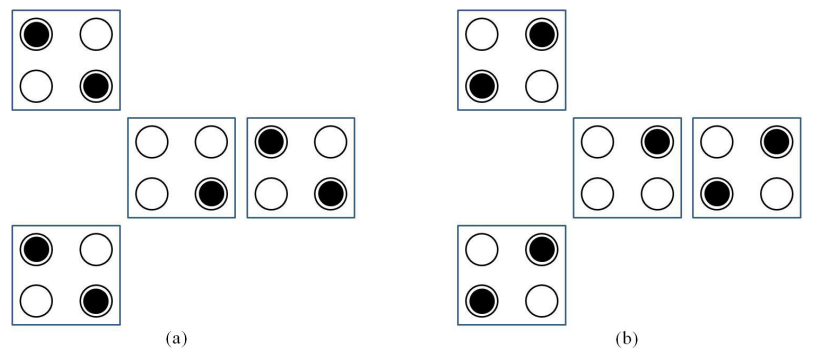

Figure 9. Output faulty cell effect on redundant inverter gate

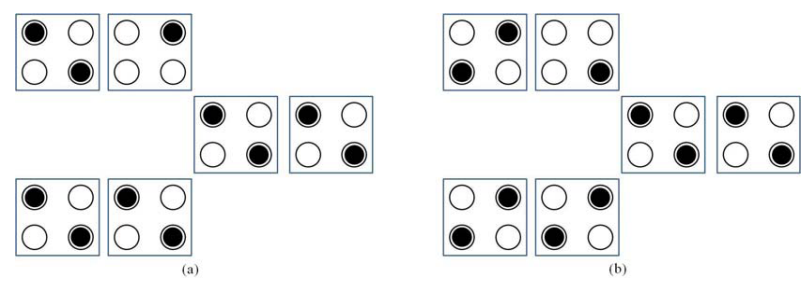

Figure 10. Up input faulty cell effect on redundant inverter gate

Table 4. Kink energy for polarization of next cell in redundant inverter gate according to the faulty cell in up or down input position

\begin{tabular}{|c|c|c|}
\hline $\begin{array}{c}\text { Input Polarization } \\
\text { and Faulty Input }\end{array}$ & $\begin{array}{c}\text { Next Cell } \\
\text { Polarization Zero }\end{array}$ & $\begin{array}{c}\text { Next Cell } \\
\text { Polarization One }\end{array}$ \\
\hline Zero and Up & 0.02313 & 0.023643 \\
\hline One and Up & 0.027216 & 0.027547 \\
\hline Zero and Down & 0.027547 & 0.027216 \\
\hline One and Down & 0.023643 & 0.02313 \\
\hline
\end{tabular}

There are also two cases for two right most cells on up and down wires. Simulation results show that if the cell in the up position is faulty, the next cell, i.e. the diagonal neighbour cell, will be stuck at zero and if the cell in the down position is faulty, the next cell, i.e. the diagonal neighbour cell, will be stuck at one. The Kink energy is computed for each polarization and the polarization with the least Kink energy 
is figured out to be the target polarization. The kink energy of each polarization is illustrated in Table 4. Figure 10 illustrates the up input faulty cell effect on redundant inverter gate.

\section{Conclusions}

Space particles such as protons, heavy ions and electrons can produce various faults when interacting with nanoelectronic devices.

A detailed modelling and characterization of single electron fault for QCA inverter gates has been represented in this paper. As stated before, the behaviour of single electron fault in QCA devices is not similar to either previously investigated faults or conventional CMOS logic. For example, stuck at zero or stuck at one fault model in redundant inverter gate is based on the input on which the fault occurs.

\section{REFERENCES}

[1] C. S. Lent, P. D. Tougaw, W. Porod, and G. H. Bernstein, "Quantum Cellular Automata," Nanotechnology, Vol. 4, No. 1, pp. 49-57, 1993.

[2] M. B. Tahoori, J. Huang, M. Momenzadeh, F. Lombardi, "Testing of Quantum Cellular Automata," IEEE Trans. on Nanotechnology, Vol. 3, No. 4, pp. 432-442, Dec. 2004.

[3] M. Momenzadeh, M. B. Tahoori, J. Huang, F. Lombardi, "Quantum Cellular Automata: New Defects and Faults for New Devices," in 18th International Parallel and Distributed Processing Symposium, 2004.

[4] M. Momenzadeh, J. Huang, F. Lombardi, "Defect Characterization and Tolerance of QCA Sequential Devices and Circuits," in 20th IEEE International Symposium on Defect and Fault Tolerance in VLSI Systems, 2005.

[5] M. Momenzadeh, J. Huang, M. B. Tahoori, F. Lombardi, "Characterization, Test, and Logic Synthesis of And-Or-Inverter (AOI) Gate Design for QCA Implementation," IEEE Trans. on Computer-Aided Design of
Integrated Circuits and Systems, Vol. 24, No. 12, pp. 1881 1893, Dec. 2005.

[6] J. Huang, M. Momenzadeh, M. B. Tahoori, F. Lombardi, "Defect Characterization for Scaling of QCA Devices," in 19th IEEE International Symposium on Defect and Fault Tolerance in VLSI Systems, 2004.

[7] P. Gupta, N. K. Jha, L. Lingappan, "A Test Generation Framework for Quantum Cellular Automata Circuits," IEEE Trans. on VLSI Systems, Vol. 15, No. 1, pp. 24-36, Jan. 2007.

[8] M. Mahdavi, M. A. Amiri, S. Mirzakuchaki, "SEU Effects on QCA Circuits," Proceedings of International Conference on Test and Diagnosis, China, Apr. 2009.

[9] P. D. Tougaw and C. S. Lent, "Dynamic Behavior of Quantum Cellular Automata," J. Appl. Phys., Vol. 80, No. 8, pp. 4722-4735, Oct. 1996.

[10] P. D. Tougaw, C. S. Lent, and W. Porod, "Bistable Saturation in Coupled Quantum-dot Cells," J. Appl. Phys., Vol. 74, No. 5, pp. 3558-3565, Sep. 1993.

[11] P.D. Tougaw and C.S. Lent, "Logical Devices Implemented Using Quantum Cellular Automata," J. Appl. Phys., Vol. 75(3), pp. 1818-1825, 1994.

[12] M. A. Amiri, M. Mahdavi, S. Mirzakuchaki, "QCA Implementation of a Mux-Based FPGA CLB," in International Conference On Nanoscience and Nanotechnology, Australia, Feb. 2008, pp. 141-144.

[13] K. Hennessy and C. S. Lent, "Clocking of Molecular Quantum-dot Cellular Automata," J. Vac. Sci. Technol., Vol. 19, No. 5, pp. 1752-1755, Sep. 2001.

[14] K. Kim, K. Wu, R. Karri, "The Robust QCA Adder Designs Using Composable QCA Building Blocks," IEEE Trans. On Computer-Aided Design of Integrated Circuits and Systems, Vol. 26, NO. 1, Jan. 2007.

[15] V. Vankamamidi, M. Ottavi, F. Lombardi, "Two-Dimensional Schemes for Clocking/Timing of QCA Circuits," IEEE Trans. on Computer-Aided Design of Integrated Circuits and Systems, Vol. 27, No. 1, pp. 34-44, Jan. 2008.

[16] Heumpil Cho and Earl E. Swartzlander, "Adder Designs and Analyses for Quantum-Dot Cellular Automata," IEEE Trans. on Nanotechnology, vol. 6, No. 3, pp. 374-383, May. 2007. 